\title{
Snapshots of the Fluctuating Hydrogen Bond Network in Liquid Water on the Sub-Femtosecond Timescale with Vibrational Resonant Inelastic x-ray Scattering
}

\author{
A. Pietzsch, ${ }^{1,{ }^{*}}$ F. Hennies, ${ }^{2}$ P. S. Miedema, ${ }^{1}$ B. Kennedy, ${ }^{1}$ J. Schlappa,${ }^{1}$ T. Schmitt, ${ }^{3}$ V. N. Strocov, ${ }^{3}$ and A. Föhlisch ${ }^{1,4}$ \\ ${ }^{1}$ Institute for Methods and Instrumentation in Synchrotron Radiation Research G-ISRR, \\ Helmholtz-Zentrum für Materialien und Energie GmbH, Albert-Einstein-Strasse 15, 12489 Berlin, Germany \\ ${ }^{2}$ MAX IV Laboratory, Box 118, 22100 Lund, Sweden \\ ${ }^{3}$ Swiss Light Source, Paul Scherrer Institut, CH-5232 Villigen PSI, Switzerland \\ ${ }^{4}$ Fakultät für Physik und Astronomie, Universität Potsdam, Karl-Liebknecht-Strasse 24-25, 14476 Potsdam, Germany
}

(Received 19 August 2014; published 24 February 2015)

\begin{abstract}
Liquid water molecules interact strongly with each other, forming a fluctuating hydrogen bond network and thereby giving rise to the anomalous phase diagram of liquid water. Consequently, symmetric and asymmetric water molecules have been found in the picosecond time average with IR and optical Raman spectroscopy. With subnatural linewidth resonant inelastic x-ray scattering (RIXS) at vibrational resolution, we take sub-femtosecond snapshots of the electronic and structural properties of water molecules in the hydrogen bond network. We derive a strong dominance of nonsymmetric molecules in liquid water in contrast to the gas phase on the sub-femtosecond timescale of RIXS and determine the fraction of highly asymmetrically distorted molecules.
\end{abstract}

DOI: 10.1103/PhysRevLett.114.088302

The effect of hydrogen bonds on water molecules in a liquid and the differences from those in gas phase have been debated in great detail in recent years. X-ray spectroscopy (giving access to the local electronic structure) has been extensively employed to study this effect [1-12]. Hypotheses range from structural changes [13] over broken bonds $[9,14,15]$ to motifs with different hydrogen bond geometries $[1,3,8,11,12]$.

Starting from water ice, the simplest model of the hydrogen bond network is that of tetrahedral coordination with four equivalent hydrogen bonds (two donor and two acceptor bonds) [16-18]. However, we know that the bond strength of both covalent and hydrogen bonds in liquid water fluctuates on timescales shorter than 100 fs [19], the $\left[\mathrm{H}_{2} \mathrm{O} \cdots \mathrm{H}_{2} \mathrm{O}\right]_{a q}$ pairs interconversing via the Zundel structure with $\left[\mathrm{OH}^{-} \cdots \mathrm{H}_{3} \mathrm{O}^{+}\right]_{a q}$ pairs. The interchange takes place by rearrangement of the covalent and hydrogen bonds and leads to a transfer of the proton charge without moving its mass (Grotthuss mechanism [20]). While the average hydrogen bond interaction is far weaker than the energy required for breaking a covalent $\mathrm{O}-\mathrm{H}$ bond, the collective fluctuations in the hydrogen bond network create random microenvironments suitable for bond breaking [21].

Since IR and optical Raman spectroscopies always measure a time average, a technique accessing ultrafast timescales as well as local information is needed to follow the dynamics of charge separation. In this work, with subnatural linewidth resonant inelastic $\mathrm{x}$-ray scattering (RIXS) at vibrational resolution, we can access the sofar inaccessible state of liquid water on a sub-femtosecond timescale using the SAXES spectrometer [22] at the
PACS numbers: 82.53.Uv, 33.15.Mt, 33.20.Rm, 61.25.Em

ADRESS beamline (SLS) [23]. The overall energy resolution for the RIXS experiment was less than $50 \mathrm{meV}$. Liquid-phase measurements at room temperature were facilitated using a flow cell with a 100-nm-thick diamondlike window coated with $10 \mathrm{~nm}$ Au on the inside. The liquid water sample was degassed for $30 \mathrm{~min}$ in an ultrasonic bath prior to measurements to get rid of excess oxygen gas solvated in the liquid. The liquid in the interaction region was renewed at a rate of $15 \mathrm{~Hz}$. To minimize possible window contamination, we did not expose any spot on the window longer than $20 \mathrm{~min}$ to radiation. Longer exposures did not result in noticeable differences in the spectrum. Incoming and outgoing radiation passed through the same window, both at an angle of $45^{\circ}$; the scattering angle was $90^{\circ}$. The energy scale was set with the help of $\mathrm{O}_{2}$ gas: From the resonance energy in the x-ray absorption spectrum, we obtain the absolute energy (highest resonance $\nu=4$ of the $\mathrm{O}_{2} \pi^{*}$ [24]), while the energy dispersion in RIXS is derived from the well-known $\mathrm{O}_{2}$ vibrational progression $[25,26]$.

As shown in Fig. 1, RIXS locally probes the electronic structure in an atom-specific and chemically selective way: An oxygen atom is excited into an intermediate core excited state (mapped using x-ray absorption spectroscopy). After the scattering duration time $\Delta t$, the atom decays back into the ground state, thereby also populating higher vibrational states and hence mapping the local oxygen ground state potential energy surface [Fig. 1(a)]. This process happens on the $4 \mathrm{fs}$ lifetime of the intermediate $4 a_{1}$ state and is thus much faster than IR and optical Raman measurements, allowing ultrafast probing of the potential energy surfaces of liquid water molecules. 


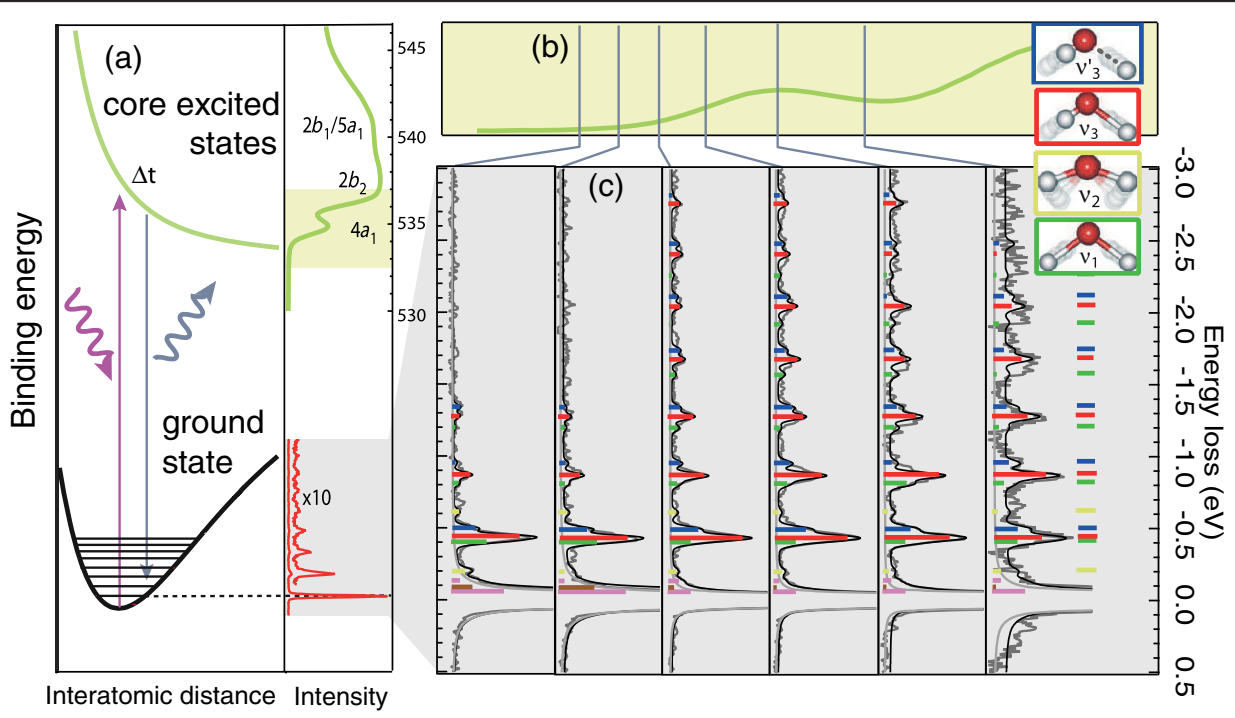

FIG. 1 (color online). Vibrational resolved RIXS of oxygen in liquid water. (a) Schematic of the RIXS process: An oxygen core electron is excited into the $4 a_{1}$ state, decaying back after the scattering duration time $\Delta t$, thereby populating several vibrational excited states in the ground state. Excitation at different energies across the $4 a_{1}$ resonance marked in the absorption spectrum blowup in (b) results in the spectra shown in (c). The main vibrational modes and librations of liquid water are fitted to the spectra, the asymmetric stretch $\nu_{3}$ (red) dominating followed by $\nu_{1}$ (symmetric stretch, green) and $\nu_{2}$ (bend, yellow). The librations (pink and brown) contribute only to a low-energy tail at the elastic peak. The blue sticks are assigned to stretch vibrations in a broken-bond molecule still localized in the hydrogen bond network.

By detuning the excitation energy from the resonance, we can shorten $\Delta t$ further as wave-packet evolution within the intermediate state is suppressed [27].

The profile of the vibrational peaks gets broader with larger energy loss. The increased broadness of these peaks indicates that the peak consists of more than one vibrational mode with close-lying energies. The peak maximum positions, however, correspond to those of the asymmetric stretch mode $\nu_{3}$ of liquid water [28] [red sticks in Fig. 1(c)]. Fitting the vibrational progression with the vibrational modes $\nu_{1}, \nu_{2}$, and $\nu_{3}$ and librations $L_{1}$ and $L_{2}$ of liquid water [28-30] gives good agreement with the experiment; see Fig. 1(c.) However, we observe the presence of an additional mode $\nu_{3}^{\prime}$ which we assign to $\mathrm{H}-\mathrm{O} \cdots \mathrm{H}$ molecules where one $\mathrm{O}-\mathrm{H}$ bond lost its covalency due to bond elongation. As the hydrogen bond network is still intact, this situation resembles the onset of the Grotthuss mechanism, where charges separate in the water network.

From the energies and intensities of the vibrational peaks, we can now obtain information on the water molecule in its electronic ground state: While the energy losses map the ground state potential energy surface [26], the intensities depend on the intermediate core excited state properties and dynamics [31]. A certain nuclear motion preferentially driven in the intermediate state affects the intensities of the vibrational overtones in the electronic ground state which then is a signature for the molecular structure. In the case of water, in initially symmetric molecules, excitation into the $4 a_{1}$ state excites the $\nu_{1}$ and $\nu_{3}$ with similar probability while initially asymmetric molecules have a higher probability to be excited asymmetrically due to the different covalent bond strengths.

In Fig. 2(b), we compare the intensity percentage of the asymmetric stretch $\nu_{3} /\left(\nu_{1}+\nu_{3}+\nu_{3}^{\prime}\right)$ and the symmetric stretch $\nu_{1} /\left(\nu_{1}+\nu_{3}+\nu_{3}^{\prime}\right)$ as a function of detuning from the $4 a_{1}$ resonance (and thus decreasing $\Delta t$ ). We find twice as much intensity for $\nu_{3}$ already at $\Delta t=0.3 \mathrm{fs}$, meaning that this ratio difference cannot be the sole result of excitation-induced nuclear motion but rather images a fraction of initially asymmetric molecules, supporting a nonuniform molecular structure at these timescales.

When following the dynamics by varying the detuning, we find that the fraction of asymmetric molecules increases for detuning from $-1.0 \mathrm{eV}$ to $-0.75 \mathrm{eV}$, corresponding to a scattering duration time of $\Delta t=0.3-0.4 \mathrm{fs}$. At the same time, the fraction of symmetric molecules decreases. This is due to the number of core excitations of the oxygen atom increasing with longer $\Delta t$ which (following the $Z+1$ approximation which takes into account that, by removing the core electron, the effective core charge of oxygen is increased by 1) creates $\mathrm{H}_{2} \mathrm{~F}^{+}$-like molecules where preferentially one $\mathrm{O}-\mathrm{H}$ bond is driven to form an asymmetric HF-like molecule. Symmetric molecules become asymmetric in that course of events while asymmetry is further enhanced in the asymmetric ones, eventually leading to one $\mathrm{OH}$ bond stretched too far to still be called a covalent $\mathrm{OH}$ bond. However, since the hydrogen of the stretched bond is still firmly anchored in the hydrogen bond network, this process does not generate ionic $\mathrm{OH}^{-}$and the strongly asymmetric molecule can still be considered a water 


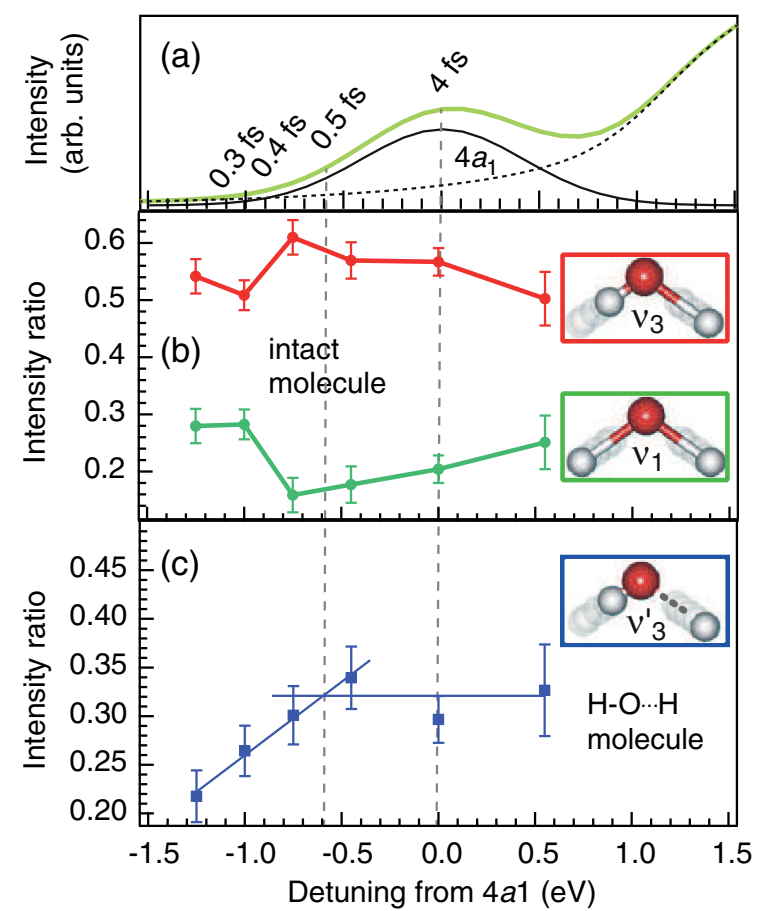

FIG. 2 (color online). (a) Enlargement of the x-ray absorption spectrum of liquid water with decomposition of contribution from $4 a_{1}$ (black straight line) and higher-lying states (dotted line). The scattering duration time is indicated for several detuning values. (b) Intensity ratios $\nu_{3} /\left(\nu_{1}+\nu_{3}+\nu_{3}^{\prime}\right)$ (fraction of asymmetric molecules, red) and $\nu_{1} /\left(\nu_{1}+\nu_{3}+\nu_{3}^{\prime}\right)$ (fraction of symmetric molecules, green) as a function of detuning from the $4 a_{1}$ resonance. The highest contribution from asymmetric molecules is evident at $-0.75 \mathrm{eV}$ detuning. (c) Intensity ratio $\nu_{3}^{\prime} /\left(\nu_{1}+\nu_{3}\right)$ (fraction of $\mathrm{H}-\mathrm{O} \cdots \mathrm{H}$ molecules vs intact molecules) as a function of detuning from the $4 a_{1}$ resonance. The ratio's increase rate flattens out after $\approx 0.5 \mathrm{fs}$. The error bars are from the fit.

molecule (further denoted $\mathrm{H}-\mathrm{O} \cdots \mathrm{H}$ ). The probability for electron delocalization increases in these molecules. This is observed as a decrease in the asymmetric percentage in Fig. 2(b) after $\approx 0.5$ fs as atoms with delocalized electrons cannot decay through the participator channel (the decay channel in which the x-ray excited electron itself fills the core hole).

Investigating the dynamics of $\mathrm{H}-\mathrm{O} \cdots \mathrm{H}$ molecules with respect to intact molecules, we plot the ratio $\nu_{3}^{\prime} /\left(\nu_{1}+\nu_{3}\right)$ in Fig. 2(c). It increases with longer $\Delta t$ as the oxygen core excitation drives molecular asymmetry further, creating more $\mathrm{H}-\mathrm{O} \cdots \mathrm{H}$ from initially (due to the hydrogen bond network) highly asymmetric molecules. However, a certain degree of initial asymmetry is the prerequisite for creation of $\mathrm{H}-\mathrm{O} \cdots \mathrm{H}$. Core excitation alone increases molecular asymmetry but cannot drive the $\mathrm{O}-\mathrm{H}$ bond far enough during the core hole lifetime to lose covalency.

At a $\Delta t \approx 0.5 \mathrm{fs}$, at the same time when electron delocalization starts to become more important, the ratio becomes constant: All initially highly asymmetric molecules have turned into $\mathrm{H}-\mathrm{O} \cdots \mathrm{H}$ molecules. This gives us the timescale on which the Grotthuss mechanism in liquid water occurs: The cleavage of an oxygen-hydrogen bond happens within $0.5 \mathrm{fs}$ after excitation. Here we initiate the bond breaking by creating a core hole, but the timescale is also true for fluctuations in liquid water, where the covalent bond is broken spontaneously. From the fraction of $\mathrm{H}-\mathrm{O} \cdots \mathrm{H}$ molecules at long $\Delta t$, we obtain the estimate of $1 / 3$ of $\mathrm{H}-\mathrm{O} \cdots \mathrm{H}$ molecules with respect to intact molecules, corresponding to $25 \%$ initially highly asymmetric molecules and thus the maximum fraction of molecules to undergo spontaneous ionization. This is found to be in agreement with the computational model of Kühne and Khaliullin [13].

Electron delocalization for RIXS via itinerate states strongly suppresses the participator channel and sets in above the $4 a_{1}$ resonance. Still, there is a signature of the structurally distorted $\mathrm{H}-\mathrm{O} \cdots \mathrm{H}$ molecules. Tuning the excitation energy across the $4 a_{1}$ resonance, the oxygen lone pair $1 b_{1}$ changes from a single peak into a double structure $1 b_{1}^{\prime \prime}$ and $1 b_{1}^{\prime}$, as shown in Fig. 3.

The origin of a splitting in the $1 b_{1}$ lone-pair region has been subject of controversy: On the one hand, it has been attributed to an intrinsic ground-state feature of liquid water where two distinct structural motifs (denoted high- and low-density liquid water) coexist $[8,11]$ and nuclear dynamics only contributes marginally [32]. On the other hand, the splitting has been assigned to a pure dynamical effect resulting from nuclear motion after core excitation $[9,14,15]$. Both interpretations have been supported and challenged [32-35] and no consensus seems to have been reached.

We observe that the splitting starts to appear at the same time as the $\mathrm{H}-\mathrm{O} \cdots \mathrm{H}$ fraction becomes constant. The intensity ratio of the $1 b_{1}^{\prime}$ and the $1 b_{1}^{\prime \prime}$ is constant across the $4 a_{1}$, mirroring the ratio $\nu_{3}^{\prime} /\left(\nu_{1}+\nu_{3}\right)$. Excitation at the main resonance and above shows different (but still constant) intensity ratios as core excitation into different states drives molecular asymmetry with different strength.

We investigate the molecular asymmetry upon core excitation and its implication on the spectra with a simple model: We used the density functional theory (DFT) ORCA program [36] applying the BP86 potential and the def2TZVP basis set [37] for all atoms to calculate nonresonant $\mathrm{x}$-ray emission spectra (XES). First, geometry optimizations were performed on a simple model: eight molecules of water having two "central" water molecules, i.e., two molecules having both two hydrogen atoms donating hydrogen bonding as well as the oxygen atom accepting from two different hydrogen atoms hydrogen bonding. One of the central water molecules is used as an internal reference, while for the other central water molecule, one $\mathrm{O}-\mathrm{H}$ bond is shortened without further geometry optimization. In the nonresonant XES calculations of the geometry optimized set of eight water molecules and of the sets with the asymmetric central water molecules, the 


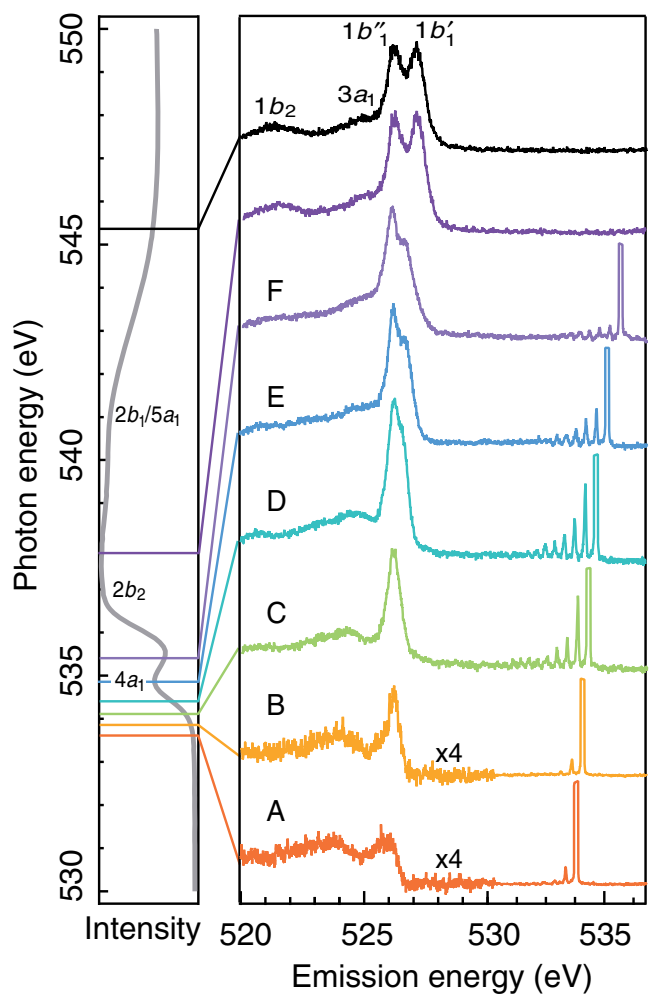

FIG. 3 (color online). RIXS spectra of liquid water measured at different excitation energies marked in the left panel. The spectra are normalized to the height of the elastic peak. Vibrations in the ground state are only observed for excitation on the $4 a_{1}$ resonance, since excitation at higher energies results in delocalization of the electron. The $1 b_{1}$ peak splits up after 0.5 fs scattering duration time (spectrum $D$ ), at the same scattering duration time as electron delocalization becomes important.

proper O 1s molecular orbitals are selected and the XES calculations are performed in similar fashion as described in Ref. [38]. The peak energy of the $1 b_{1}$ shifts almost linearly to lower energies with increased molecular asymmetry, as shown in Fig. 4(b). The appearance of the $1 b_{1}$ peak can thus be considered linked to the molecular asymmetry and the concomitant loss of covalency in the elongated $\mathrm{O} \cdots \mathrm{H}$ bonds. How this results in two peaks rather than an asymmetrically broadened peak is shown in Fig. 4(c), where qualitative potential energy curves of the intermediate and valence hole final state are plotted. The final state shows two separate minima, one for the covalently bonded $\mathrm{H}$ and one for the hydrogen bonded $\mathrm{H}$, the potential barrier height between them depending on the hydrogen bond strength. For short $\Delta t$, the core excited state can only decay into the covalent bond minimum at $r_{1}=1 \AA$. For longer $\Delta t$, the $\mathrm{O}-\mathrm{H}$ bond can elongate significantly, allowing also decay into the hydrogen bond final state minimum and thus giving rise to the double peak structure.

The splitting of the $1 b_{1}$ lone pair peak is thus found to be a result from an intrinsic ground state feature (the molecular asymmetry due to the hydrogen bond network) combined

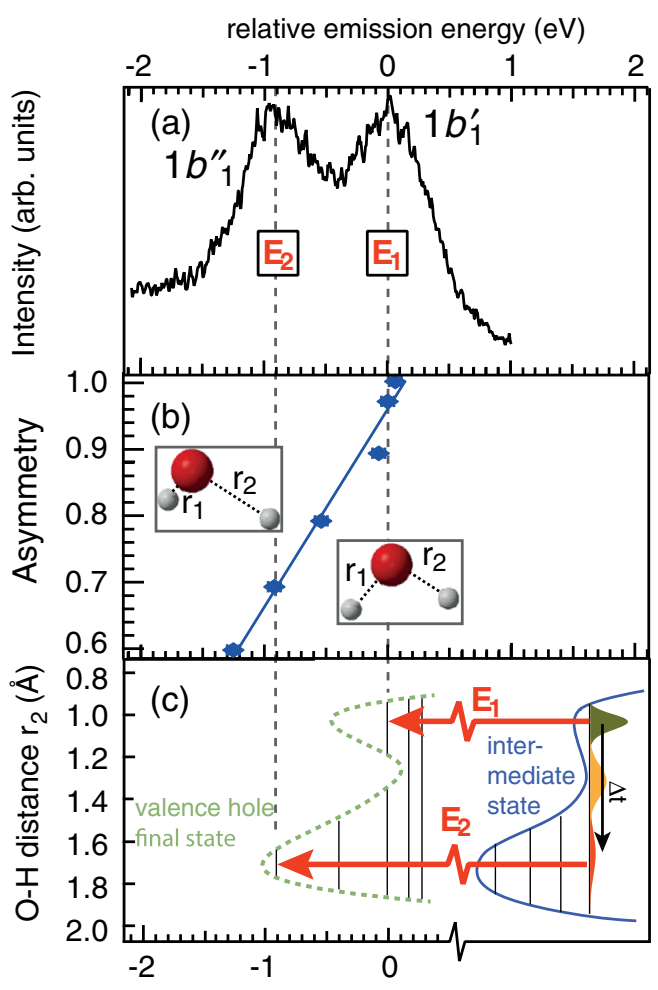

FIG. 4 (color online). The oxygen lone pair split peak. (a) Experimental $1 b_{1}$ double peak shift for nonresonant excitation. (b) DFT calculations give different peak shifts for asymmetric molecules in dependence of the molecular asymmetry ratio $r_{1} / r_{2}$. Using this simple model, the $1 b_{1}^{\prime \prime}$ peak corresponds to a molecular asymmetry of 0.69 . (c) Qualitative potential energy curves of the intermediate and valence hole final state. In the proposed mechanism, the $1 b_{1}$ peak splitting corresponds to the difference of $E_{1}$ and $E_{2}$.

with nuclear dynamics after core excitation. This dynamics dominates as has also been seen for hydrogen bonding in liquid alcohols [39].

In the case of gas phase water [35], the $1 b_{1}$ split peak is also present, but only for x-ray excitation to the $4 a_{1}$ unoccupied state. This means that only the strongly antibonding $4 a_{1}$ elongates the $\mathrm{O}-\mathrm{H}$ bond far enough to give rise to the $1 b_{1}^{\prime \prime}$ peak. All other excitations in gas phase water result in ionization rather than stretching of the $\mathrm{O}-\mathrm{H}$ bond. In the liquid, the hydrogen bond network induces molecular asymmetry which even dominates dynamics driven by excitation above the $4 a_{1}$, as seen in the double peak's presence at the $4 a_{1}$ and above.

In summary, we established with unprecedented resolution the fraction of asymmetric molecules in liquid water through sub-femtosecond snapshots and we interpret these fully with independently established theory [13]. These findings also give a fresh look at a controversy more than a decade long, in relation to low-resolution RIXS spectral signatures. We find that in addition to the fraction of asymmetric molecules, strong dynamics in the core excited state dominate these features. 
We thank J.-E. Rubensson and U. Schade for fruitful discussions, J. Andersson for providing the carbon membrane windows, and $\mathrm{K}$. Zhou for technical support. The experiments were performed at the ADRESS beamline of the Swiss Light Source at the Paul Scherrer Institut. We acknowledge support by the Swedish Research Council (VR) and Carl Tryggers Stiftelse (CTS). The research leading to these results has received funding from the European Community's Seventh Framework Programme (FP7/2010-2011) under Grant Agreement No. 226716 (for ELISA).

*annette.pietzsch@helmholtz-berlin.de

[1] P. Wernet, D. Nordlund, U. Bergmann, M. Cavalleri, M. Odelius, H. Ogasawara, L. A. Näslund, T. K. Hirsch, L. Ojamäe, P. Glatzel, L. G. M. Pettersson, and A. Nilsson, Science 304, 995 (2004).

[2] J. D. Smith, C. D. Cappa, K. R. Wilson, B. M. Messer, R. C. Cohen, and R. J. Saykally, Science 306, 851 (2004).

[3] A. Nilsson, D. Nordlund, I. Waluyo, N.Huang, H. Ogasawara, S. Kaya, U. Bermann, L.-Å. Näslund, H. Öström, P. Wernet, K. J. Andersson, T. Schiros, and L. G. M. Petterson, J. Electron Spectrosc. Relat. Phenom. 177, 99 (2010).

[4] B. Winter, U. Hergenhahn, M. Faubel, O. Björneholm, and I. V. Hertel, J. Chem. Phys. 127, 094501 (2007).

[5] D. Nordlund, H. Ogasawara, H. Bluhm, O. Takahashi, M. Odelius, M. Nagasono, L. G. M. Pettersson, and A. Nilsson, Phys. Rev. Lett. 99, 217406 (2007).

[6] J.-H. Guo, Y. Luo, A. Augustsson, J.-E. Rubensson, C. Såthe, H. Ågren, H. Siegbahn, and J. Nordgren, Phys. Rev. Lett. 89, 137402 (2002).

[7] M. Odelius, H. Ogasawara, D. Nordlund, O. Fuchs, L. Weinhardt, F. Maier, E. Umbach, C. Heske, Y. Zubavichus, M. Grunze, J. D. Denlinger, L. G. M. Pettersson, and A. Nilsson, Phys. Rev. Lett. 94, 227401 (2005).

[8] T. Tokushima, Y. Harada, O. Takahashi, Y. Senba, H. Ohashi, L. G. M. Petterson, A. Nilsson, and S. Shin, Chem. Phys. Lett. 460, 387 (2008).

[9] O. Fuchs, M. Zharnikov, L. Weinhardt, M. Blum, M. Weigand, Y. Zubavichus, M. Bär, F. Maier, J. D. Denlinger, C. Heske, M. Grunze, and E. Umbach, Phys. Rev. Lett. 100, 027801 (2008).

[10] K. M. Lange, R. Könnecke, S. Ghadimi, R. Golnak, M. A. Soldatov, and E. F. Aziz, Chem. Phys. 377, 1 (2010).

[11] A. Nilsson, T. Tokushima, Y. Horikawa, Y. Harada, M. P. Ljungberg, S. Shin, and L. G. M. Pettersson, J. Electron Spectrosc. Relat. Phenom. 188, 84 (2013).

[12] Y. Harada, T. Tokushima, Y. Horikawa, O. Takahashi, H. Niwa, M. Kobayashi, M. Oshima, Y. Senba, H. Ohashi, K. T. Wikfeldt, A. Nilsson, L. G. M. Pettersson, and S. Shin, Phys. Rev. Lett. 111, 193001 (2013).

[13] T. D. Kühne and R. Z. Khaliullin, Nat. Commun. 4, 1450 (2013).
[14] M. Odelius, Phys. Rev. B 79, 144204 (2009).

[15] M. Odelius, J. Phys. Chem. A 113, 8176 (2009).

[16] J. D. Bernal and R. H. Fowler, J. Chem. Phys. 1, 515 (1933).

[17] F. H. Stillinger, Science 209, 451 (1980).

[18] G. N. I. Clark, C. D. Cappa, J. D. Smith, R. J. Saykally, and T. Head-Gordon, Mol. Phys. 108, 1415 (2010).

[19] S. Woutersen and H. J. Bakker, Phys. Rev. Lett. 96, 138305 (2006).

[20] S. Cukierman, Biochim. Biophys. Acta 1757, 876 (2006).

[21] P. L. Geissler, C. Dellago, D. Chandler, J. Hutter, and M. Parrinello, Science 291, 2121 (2001).

[22] G. Ghiringhelli, A. Piazzalunga, C. Dallera, G. Trezzi, L. Braicovich, T. Schmitt, V. N. Strocov, R. Betemps, L. Patthey, X. Wang, and M. Grioni, Rev. Sci. Instrum. 77, 113108 (2006).

[23] V. N. Strocov, T. Schmitt, U. Flechsig, T. Schmidt, A. Imhof, Q. Chen, J. Raabe, R. Betemps, D. Zimoch, J. Krempasky, X. Wang, M. Grioni, A. Piazzalunga, and L. Patthey, J. Synchrotron Radiat. 17, 631 (2010).

[24] K. C. Prince, R. Richter, M. de Simone, M. Aglaia, and M. Coreno, J. Phys. Chem. A 107, 1955 (2003).

[25] A. S.-C. Cheung, K. Yoshino, J. R. Esmond, and W. H. Parkinson, J. Mol. Spectrosc. 178, 66 (1996).

[26] F. Hennies, A. Pietzsch, M. Berglund, A. Föhlisch, T. Schmitt, V. N. Strocov, H. O. Karlsson, J. Andersson, and J.-E. Rubensson, Phys. Rev. Lett. 104, 193002 (2010).

[27] F. Gel'mukhanov, T. Privalov, and H. Ågren, Phys. Rev. A 56, 256 (1997).

[28] G. E. Walrafen and E. Pugh, J. Solution Chem. 33, 81 (2004).

[29] A. A. Yakovenko, V. A. Yashin, A. E. Kovalev, and E. E. Fesenko, Biophysics 47, 891 (2002).

[30] R. M. Pope and R. S. Fry, Appl. Opt. 36, 8710 (1997).

[31] A. Pietzsch, Y.-P. Sun, F. Hennies, Z. Rinkevicius, H. O. Karlsson, T. Schmitt, V. N. Strocov, J. Andersson, B. Kennedy, J. Schlappa, A. Föhlisch, J.-E. Rubensson, and F. Gelmukhanov, Phys. Rev. Lett. 106, 153004 (2011).

[32] M. P. Ljungberg, L. G. M. Pettersson, and A. Nilsson, J. Chem. Phys. 134, 044513 (2011).

[33] J. Forsberg, J. Gråsjö, B. Brena, J. Nordgren, L.-C. Duda, and J.-E. Rubensson, Phys. Rev. B 79, 132203 (2009).

[34] K. M. Lange, M. Soldatov, R. Golnak, M. Gotz, N. Engel, R. Könnecke, J.-E. Rubensson, and E. F. Aziz, Phys. Rev. B 85, 155104 (2012).

[35] L. Weinhardt, A. Benkert, F. Meyer, M. Blum, R. G. Wilks, W. Yang, M. Bär, F. Reinert, and C. Heske, J. Chem. Phys. 136, 144311 (2012).

[36] F. Neese, Comput. Mol. Sci. 2, 73 (2012).

[37] F. Weigend and R. Ahlrichs, Phys. Chem. Chem. Phys. 7, 3297 (2005).

[38] C. J. Pollock and S. deBeer, J. Am. Chem. Soc. 133, 5594 (2011).

[39] S. Schreck, A. Pietzsch, K. Kunnus, B. Kennedy, W. Quevedo, P. Miedema, P. Wernet, and A. Föhlisch, Struct. Dyn. 1, 054901 (2014). 